\title{
Diffuse Large B-Cell Lymphoma
}

\author{
Norbert Schmitz, Matthias Stelljes, \\ and Ali Bazarbachi
}

\subsection{Definition and Epidemiology}

Diffuse large B-cell lymphoma (DLBCL) is a neoplasm of morphologically medium to large B-lymphoid cells. The most recent WHO classification of tumors of hematopoietic and lymphoid tissues (Swerdlow et al. 2017) divides DLBCL into DLBCL, NOS with distinct morphological (centroblastic, immunoblastic, anaplastic, rare) and molecular (germinal center B-cell, activated B-cell) subtypes, other lymphomas of large B cells, high-grade B-cell lymphoma (with MYC and BCL2 and/or BCL6 rearrangements or NOS), and B-cell lymphoma, unclassifiable.

With some important exceptions, diagnostic work-up and treatment are identical in all DLBCL subtypes. It is beyond the scope of this article to fully describe the exceptions; we mention the most important differences but otherwise focus

N. Schmitz $\cdot$ M. Stelljes

Department of Medicine A, Hematology, Oncology, Pneumonology and Hemostaseology, University Hospital Münster, Münster, Germany

A. Bazarbachi $(\bowtie)$

Bone Marrow Transplantation Program, Department of Internal Medicine, American University of Beirut Medical Center, Beirut, Lebanon

e-mail: bazarbac@aub.edu.lb on transplantation for patients with relapsed/ refractory DLBCL.

DLBCL is the most frequent lymphoma subtype and accounts for approximately one third of newly diagnosed lymphoma cases worldwide. In Europe, the 10-year prevalence of DLBCL is estimated at 43.3 per 100,000 per year (Smith et al. 2015); the age-adjusted incidence rate of DLBCL reported by the US Surveillance, Epidemiology, and End Results (SEER) program is 7.14/100,000 person-years (Howlader et al. 2017). The disease is slightly more frequent in men than in women; it mostly is a disease of the elderly (median patient age beyond 60 years) but can occur also in children and adolescents.

\subsection{Diagnosis}

The diagnosis is made according to the WHO classification from a sufficiently large surgical specimen or excisional lymph node biopsy; needle biopsies are not recommended. Beyond morphological evaluation by an experienced pathologist, determination of the immunophenotype of the malignant cells (positivity of malignant cells for CD19 and CD20 must be documented because of its therapeutic consequences) and determination of the cell of origin by adequate molecular methods are required (Swerdlow et al. 2017). 


\subsection{Classification}

The large B-cell lymphomas comprise the morphological and molecular subtypes of DLBCL, NOS, high-grade B-cell lymphomas, and B-cell lymphomas, unclassifiable (see paragraph 1 ).

The WHO classification describes 12 other (and 1 provisional) lymphomas of large B cells. Among these, primary diffuse large B-cell lymphoma of the CNS (PCNSL), lymphomatoid granulomatosis (LG), primary mediastinal large B-cell lymphoma (PMBCL), and plasmablastic lymphoma not only show significant differences in pathogenesis and clinical manifestation but in most centers are treated different from classical DLBCL. Exact subtyping of diagnostic specimens taking into account their origin (e.g., primary cutaneous DLBCL, leg type; PCNSL; primary effusion lymphoma) is important. In order to fulfill all WHO requirements, the cell of origin (GCB- or ABC-subtype by IHC or gene expression profiling) and the presence/absence of distinct chromosomal translocations (BCL6, BCL2, MYC by FISH testing or IHC) must be determined.

\subsection{Risk Factors}

The International Prognostic Index (IPI) remains the most important tool in order to estimate the prognosis of patients with DLBCL (Ziepert et al. 2010). The IPI takes into account five factors (age, stage, LDH, performance status, and number of extranodal sites involved). Patients within the low (IPI 0, 1), low-intermediate (IPI 2), highintermediate (IPI 3), and high-risk group (IPI 4, 5) can expect 3-year overall survival of $91.4 \%, 80.9 \%$, $65.1 \%$, and $59.0 \%$, respectively, if treated with $\mathrm{R}-\mathrm{CHOP}$ or one of its variants. Other clinical risk factor models (R-IPI; NCCN-IPI) (Sehn et al. 2007; Zhou et al. 2014, respectively) have been proposed, but advantages over the original IPI seem limited.

\subsection{First-Line Treatment}

First-line treatment of patients with DLBCL generally consists of RTX at standard dose (375 mg/ sqm) in combination with CHOP (CY, DOXO,
VCR, PRD) or one of its variants such as ACVBP, CHOEP, or DA-EPOCH chemotherapy. Six cycles of R-CHOP are generally used. However, the cycle number can be reduced to four without jeopardizing treatment outcome in patients with IPI 0. Patients with early disease (IPI 0 and 1) have been treated with abbreviated chemotherapy and involved-field radiotherapy (RT). Recent studies do not support a role for RT in such patients (Lamy et al. 2018). In patients with IPI $2-5$, radiotherapy to bulky and extranodal disease is regularly recommended after R-CHOP in some but not in the majority of countries.

Several studies evaluated the role of consolidative high-dose therapy followed by autoHSCT in the RTX era. The French (Gouill et al. 2007), Italian (Chiappella et al. 2017), and German (Schmitz et al. 2012) studies failed to demonstrate an advantage of auto-HSCT over conventional chemotherapy. The only American study (Stiff et al. 2013) reported an advantage of auto-HSCT in younger patients with high-risk disease (age-adjusted IPI 3); however, this study included patients treated with CHOP only and patients with T-cell lymphoma and as a consequence was underpowered in order to show a significant advantage of auto-HSCT over R-CHOP (Schmitz et al. 2014). In young patients who remain PET positive after two cycles of chemo-immunotherapy, auto-HSCT is performed in some but not in the majority of countries.

Patients with PCNSL or DLBCL with primary involvement of testicles must receive chemotherapy penetrating into the CNS.

More aggressive chemotherapies (CHOEP, DA-EPOCH, or ACVBP) in combination with RTX with or without RT are recommended in patients with PMBCL or plasmablastic lymphoma and in patients with high-intermediate and high-risk disease (Ghielmini et al. 2013; Recher et al. 2011). If patients with DLBCL of $\mathrm{ABC}$ subtype or rearrangement of MYC and BCL2 and/or BCL6 (double- or triple-hit lymphoma) should receive more aggressive therapy, therapy remains controversial (Friedberg 2017; Staiger et al. 2017). The same holds true for the value of targeted therapies (ibrutinib, lenalidomide, and others) given in addition to 
R-CHOP. Prospective randomized studies addressing these questions have been closed but not published yet.

\subsection{Second-Line Treatment}

The principles of management of relapsed and refractory DLBCL are shown in Table 85.1. All chemotherapy-based salvage regimens cause hematologic toxicity in many cases necessitating RBC and platelet transfusions. Mucositis, gastrointestinal toxicities, neutropenic fever, and infections are reported in a significant proportion of patients. Nephrotoxicity, hepatotoxicity, and other non-hematologic toxicities are also observed. Failure to mobilize hematopoietic

Table 85.1 Management of relapsed or refractory DLBCL

- New biopsy: Highly recommended in all patients with R/R DLBCL. Core biopsies acceptable

- Radiological evaluation: PET/CT recommended for evaluation of treatment outcome

- Salvage therapy followed by auto-HSCT is currently considered standard of care for patients with R/R DLBCL. Especially in patients with refractory disease or early relapse (within 12 months from the end of first-line therapy), results are not satisfactory

- Allo-HSCT should be considered in younger patients without comorbidities especially in patients with refractory disease or early relapse. Patients relapsing after auto-HSCT are candidates for allo-HSCT

- CAR T cells are a valid option whenever available

- Selection of salvage therapy: Randomized studies failed to show significant differences in terms of efficacy or toxicity with different salvage regimens. R-DHAP seems superior to R-ICE for patients with a GCB subtype (Thieblemont et al. 2011).

Therefore, salvage strategies should take into account individual patient characteristics (age and comorbidities) considering potential cumulative hematologic and non-hematologic toxicity and the possibility of harvesting stem cells. Cardiac, pulmonary, renal, and liver function should be evaluated prior to treatment

- The objective of salvage chemotherapy is to induce a complete or partial response indicating that the tumor remains chemosensitive, this having a major impact on outcome after transplantation. PET negativity after salvage therapy is a surrogate marker of chemosensitivity and predicts patient outcome after auto-HSCT
Table 85.2 Response to salvage regimens

\begin{tabular}{|c|c|}
\hline \multicolumn{2}{|c|}{ Regimens compared in prospective randomized trials } \\
\hline $\begin{array}{l}\text { R-DHAP (Gisselbrecht } \\
\text { et al. 2010) }\end{array}$ & $\begin{array}{l}\text { Dexamethasone, } \\
\text { cytarabine, cisplatin } \\
\text { ORR } 62.8 \%(44.1 \%)^{\mathrm{a}} \text {, } \\
\text { CR } 28 \%(14.6 \%)^{\mathrm{a}}\end{array}$ \\
\hline GDP (Crump et al. 2014) & $\begin{array}{l}\text { Gemcitabine, } \\
\text { dexamethasone, cisplatin } \\
\text { ORR } 45.1 \% \text {, CR } 13.8 \% \\
\text { after } 2 \text { cycles }\end{array}$ \\
\hline $\begin{array}{l}\text { ICE (Gisselbrecht } \\
\text { et al. 2010) }\end{array}$ & $\begin{array}{l}\text { Ifosfamide, carboplatin, } \\
\text { etoposide } \\
\text { ORR } 63.5 \% \text {, CR } 24 \%\end{array}$ \\
\hline
\end{tabular}

Addition of new drugs (lenalidomide, ibrutinib, brentuximab vedotin, polatuzumab, other) to RTXchemo in order to improve response rates of salvage regimens is not recommended outside clinical trials

apercentages in brackets from Crump et al. (2014)

stem cells in $10-20 \%$ of cases occurs with all salvage regimens. Efficacy of different salvage options is shown in Table 85.2.

\subsection{Autologous HSCT}

Autologous HSCT is still considered the standard treatment for patients with refractory or relapsed (R/R) DLBCL. In the RTX era, however, the results of salvage therapy followed by auto-HSCT are less convincing than before, and the benefit of auto-HSCT even for those patients achieving PR or CR with salvage chemotherapy and RTX is limited (Crump et al. 2014, 2017). In particular, patients with refractory disease or early relapse pretreated with RTX as part of first-line therapy rarely achieve long-term remissions after auto-HSCT. In the CORAL study, 3-year PFS for such patients was only $23 \%$ although those proceeding to auto-HSCT showed 3-year PFS of $39 \%$. Alternative treatment, e.g., allo-HSCT, may be more adequate. EBMT indications (Sureda et al. 2015) for auto-HSCT in DLBCL are shown in Table 85.3. Auto-HSCT is generally not recommended as part of first-line therapy in DLBCL although recent data on PET-guided auto-HSCT are promising (Casasnovas et al. 2017). We discourage auto-HSCT for patients with refractory disease not responding to salvage therapy. 
Table 85.3 Indications for auto-HSCT in DLBCL

\begin{tabular}{|l|l|} 
Disease status & Recommendations \\
\hline First complete remission & $\begin{array}{l}\text { Clinical option } \\
\text { Level of evidence I }\end{array}$ \\
$\begin{array}{l}\text { Sensitive relapse/ } \geq \text { 2nd complete } \\
\text { response }\end{array}$ & $\begin{array}{l}\text { Standard of care } \\
\text { Level of evidence I }\end{array}$ \\
\hline Refractory disease & $\begin{array}{l}\text { Clinical option } \\
\text { Level of evidence }\end{array}$ \\
& II
\end{tabular}

\subsubsection{HSC Source}

PBSC is used in $>90 \%$ of auto-HSCT.

\subsubsection{Consolidation (High-Dose Therapy)}

Consolidation (high-dose therapy) should eliminate malignant cells with minimal impact on organ systems other than hematopoiesis. The choice of the preparative regimen varies and is based on institutional experience rather than evidence. The BEAM regimen typically consisting of BCNU (300 mg/m² $\times 1$, day-6), VP (200 mg/ $\mathrm{m}^{2}$, days -5 to -2$)$, Ara-C (200 $\mathrm{mg} / \mathrm{m}^{2}$ bid, days -5 to -2$)$, and MEL ( $140 \mathrm{mg} / \mathrm{kg} /$ day $\times 1$, days -1$)$ is the preferred regimen in EBMT centers.

Acute toxicities of BEAM include severe mucositis, nausea and vomiting, diarrhea, hepatotoxicity, nephrotoxicity, and non-infective pulmonary complications. Late toxicities include pulmonary complications such as chronic interstitial fibrosis and decrease in lung diffusion capacity $(21 \%)$, infection (30\%), metabolic syndrome (17\%), cardiovascular complications $(12 \%)$, secondary tumors $(20 \%)$, and other toxicities (20\%). The most frequent cause of NRM is subsequent malignancy (12-fold increased risk compared with the general population). Late death is also attributed to cardiac toxicity ( $2 \%)$, pulmonary complications (2\%), and other treatment-related toxicities (15\%).

Other high-dose regimens have been used sometimes because of shortage of MEL or BCNU. Recent publications suggest that the BEAC (CY) and TEAM (TT) regimens show efficacy and toxicity similar to BEAM in most if not all lymphoma subtypes (Robinson et al. 2018; Sellner et al. 2016).

\subsubsection{Prognostic Factors}

Adverse prognostic factors for auto-HSCT identified in prospective studies include early relapse within 12 months of induction therapy, prior exposure to R, secondary age-adjusted IPI, poor performance status, and involvement of two or more extranodal sites at relapse.

\subsubsection{Results of Auto-HSCT ${ }^{\mathrm{a}}$}

\begin{tabular}{|l|l|l|l|} 
& NRM & OS at 3 years & EFS at 3 years \\
$\begin{array}{l}\text { Gisselbrecht } \\
\text { et al. (2012) }\end{array}$ & $1.4 \%$ & $51 \%$ (DHAP) & 35\% (DHAP) \\
$\begin{array}{l}\text { Crump et al. } \\
\text { (2014) }\end{array}$ & NR & $\begin{array}{l}39 \% \text { (both } \\
\text { arms) }\end{array}$ & $\begin{array}{l}26 \% \text { (both } \\
\text { arms) }\end{array}$
\end{tabular}

${ }^{a}$ Results from prospective randomized studies. Differences in OS and EFS may be explained by differing patient characteristics and study design

\subsubsection{Consolidation Treatment After Auto-HSCT}

There are no data and no recommendation for consolidative therapy after auto-HSCT for DLBCL. In the CORAL study Gisselbrecht et al. (2012), RTX maintenance did not improve outcome.

\subsubsection{Tandem Transplantation}

No data from the RTX era are available.

\subsubsection{Relapse After Auto-HSCT}

Patients relapsing after auto-HSCT generally have a poor prognosis. Therapeutic options are limited. Data on new drugs specifically used post autoHSCT are not available. Results of allo-HSCT after failure of auto-HSCT are reported below. CAR T cells may be a therapeutic option; however, no more than anecdotal data are available. 


\subsection{Allogeneic HSCT}

Allo-HSCT is considered a curative treatment option for patients with DLBCL who relapse or progress after auto-HSCT. The EBMT reported on 101 such patients who had been allografted for DLBCL between 1997 and 2006; 37 patients were transplanted after MAC and 64 patients after RIC (van Kampen et al. 2011). Three-year NRM was $28.2 \%$, relapse rate was $30.1 \%$, PFS was $41.7 \%$, and OS was $53.8 \%$ after 3 years. No statistically significant differences were seen between patients transplanted after MAC or RIC or patients transplanted from MRS or MUDs.

The only prospective randomized clinical trial reported so far (Glass et al. 2014) compared GVHD prophylaxis including RTX or not after MAC and allo-HSCT for R/R DLBCL. No significant difference between patients receiving or not receiving RTX in addition to standard MMF and TAC for GVHD prophylaxis was found. OS was $52 \%$ for all 82 patients randomized with significant differences between patients transplanted from a MUD or MMUD and patients receiving or not receiving ATG. For patients transplanted from matched family donors or MUD receiving ATG, OS was $64.7 \%$. These data show that alloHSCT is a valid alternative to any other treatment for patients relapsing after failure from autoHSCT. Allo-HSCT should also be considered for patients with early relapse after first-line R-CHOP or similar.

\subsubsection{Stem Cell Source}

PBSC is the preferred stem cell source for alloHSCT. The use of haploidentical donors has somewhat increased the use of BM in some of the series.

\subsubsection{Donor Selection}

In recent years, there has been a significant increase in the use of haploidentical donors for allo-HSCT after the introduction of PT-CY.
Retrospective analyses from EBMT and CIBMTR (Kanate et al. 2016; Ghosh et al. 2016) suggest that allo-HSCT from HLA-identical family and URD or from haploidentical donors give comparable results. However, no prospective clinical trials comparing haploidentical donors versus HLA-identical siblings and MUD have been published so far.

\subsubsection{Conditioning}

RIC regimens reduce NRM after transplantation in many indications but also tend to increase RI after transplantation. Because no prospective clinical trials demonstrating the superiority of one conditioning regimen over another have been reported, the question if RIC or MAC should be preferred cannot generally be answered. Aggressive disease not completely responding to salvage therapy and high tumor are situations where MAC should strongly be considered.

\subsubsection{Prognostic Factors}

The most important adverse prognostic factor that impacts long-term outcome of patients being treated with allo-HSCT is disease status before the treatment. However, unlike the situation with autoHSCT, also patients not perfectly responding to salvage therapy, e.g., patients with minor response or stable disease, may benefit from allo-HSCT.

\subsubsection{The Use of Allo-HSCT in the Era of New Drugs and CAR T Cells}

In contrast to the situation for other lymphomas, e.g., Hodgkin's disease, new drugs have not really affected the role and positioning of allo-HSCT in patients relapsing/progressing after auto-HSCT. Lenalidomides, ibrutinib, polatuzumab, or checkpoint inhibitors with or without chemotherapy can be used to bring more patients to transplantation. None of these drugs can substitute for allo-HSCT because 
remissions are mostly transient, and no cures have been achieved.

CAR T cells (Schuster et al. 2017; Neelapu et al. 2018) are an option for patients failing an autograft for DLBCL. However, results in dayto-day routine are not yet clear, and CAR T cells are not available outside clinical trials.

\subsubsection{Results of Allo-HSCT}

\begin{tabular}{|l|l|l|}
\hline NRM & OS at 3 years & PFS at 3 years \\
\hline $25-35 \%$ & $40-60 \%$ & $30-50 \%$
\end{tabular}

\subsubsection{Disease Relapse After Allo-HSCT}

Disease relapse carries a dim prognosis. Beyond DLI, therapeutic options are few, clinical trials should be actively sought, and palliative care is a reality in many cases. Checkpoint inhibitors may be an option with mixed results and risk of GVHD.

\subsection{Therapeutic Algorithm Recommended by the Authors (See Fig. 85.1)}

\subsection{Long-Term Outcomes of Auto- and Allo-HSCT in Patients with R/R DLBCL (See Fig. 85.2)}

\section{Key Points}

- In the RTX era, auto-HSCT is generally not recommended as part of first-line therapy in DLBCL although recent data on PET-guided auto-HSCT are promising. Auto-HSCT is still the standard of care for those DLBCL patients with chemosensitive first relapse. Results of auto-HSCT might improve with better

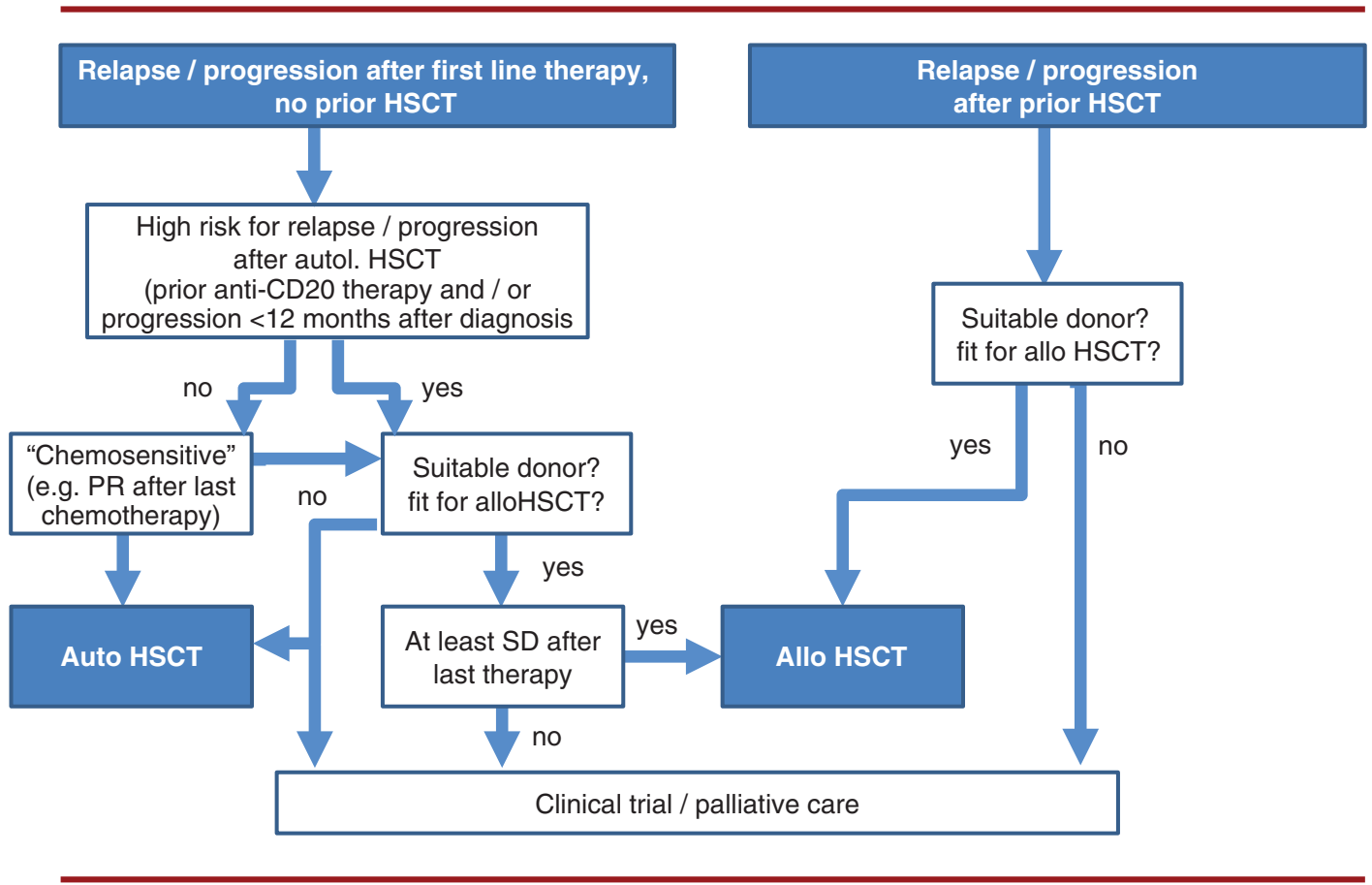

Fig. 85.1 Allogeneic and autologous HSCT in R/R lymphoma: treatment decision 
selection of patients (e.g., including PET imaging for patient selection), and improved salvage strategies results.

- Allo-HSCT is the only curative treatment option for patients with refractory disease and those relapsing after autoHSCT. Patients with early relapse $(<12$ months after first-line treatment) should be considered for allo-HSCT. Conditioning should be guided by the individual clinical situation. Haploidentical transplants may substitute for unrelated donor transplants in the near future. New drugs have not really changed the treatment algorithm for DLBCL. The role of CAR T cells is under study.
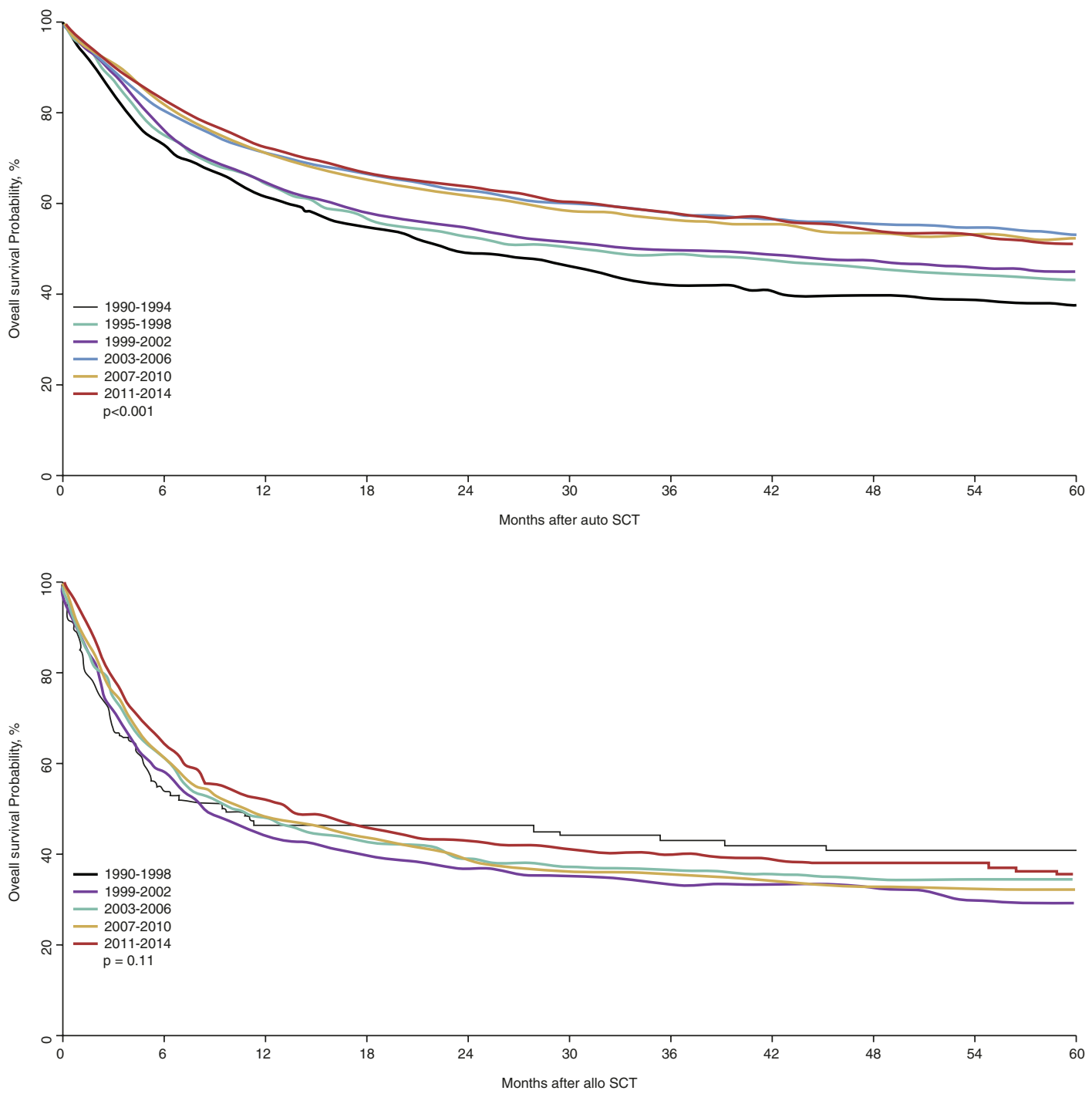

Fig. 85.2 Long-term outcomes of auto- and allo-HSCT in patients with R/R DLBCL (EBMT data base, with permission) 


\section{References}

Casasnovas RO, Ysebaert L, Thieblemont C, et al. FDGPET-driven consolidation strategy in diffuse large B-cell lymphoma: final results of a randomized phase 2 study. Blood. 2017;130:1315-26.

Chiappella A, Martelli M, Angelucci E, et al. Rituximabdose-dense chemotherapy with or without high-dose chemotherapy plus autologous stem-cell transplantation in high-risk diffuse large B-cell lymphoma (DLCL04): final results of a multicentre, open-label, randomised, controlled, phase 3 study. Lancet Oncol. 2017;18:1076-88.

Crump M, Kuruvilla J, Couban S, et al. Randomized comparison of gemcitabine, dexamethasone, and cisplatin versus dexamethasone, cytarabine, and cisplatin chemotherapy before autologous stem-cell transplantation for relapsed and refractory aggressive lymphomas: NCIC-CTG LY.12. J Clin Oncol. 2014;32:3490-6.

Crump M, Neelapu SS, Farooq U, et al. Outcomes in refractory diffuse large B-cell lymphoma: results from the international SCHOLAR-1 study. Blood. 2017;130:1800-8.

Friedberg JW. How I treat double-hit lymphoma. Blood. 2017;130:590-6.

Ghielmini M, Vitolo U, Kimby E, et al. ESMO Guidelines consensus conference on malignant lymphoma 2011 part 1: diffuse large B-cell lymphoma (DLBCL), follicular lymphoma (FL) and chronic lymphocytic leukemia (CLL). Ann Oncol. 2013;24:561-76.

Ghosh N, Karmali R, Rocha V, et al. Reduced-intensity transplantation for lymphomas using haploidentical related donors versus HLA-matched sibling donors: a Center for International Blood and Marrow Transplant Research Analysis. J Clin Oncol. 2016;34:3141-9.

Gisselbrecht C, Glass B, Mounier N, et al. Salvage regimens with autologous transplantation for relapsed large B-cell lymphoma in the rituximab era. J Clin Oncol. 2010;28:4184-90.

Gisselbrecht C, Schmitz N, Mounier N, et al. Rituximab maintenance therapy after autologous stem-cell transplantation in patients with relapsed CD20 (+) diffuse large B-cell lymphoma: final analysis of the collaborative trial in relapsed aggressive lymphoma. J Clin Oncol. 2012;30:4462-9.

Glass B, Hasenkamp J, Wulf G, et al. Rituximab after lymphoma-directed conditioning and allogeneic stemcell transplantation for relapsed and refractory aggressive non-Hodgkin lymphoma (DSHNHL R3): an open-label, randomised, phase 2 trial. Lancet Oncol. 2014;15:757-66.

Gouill SL, Milpied N, Buzyn A, et al. Allogeneic stem cell transplantation (allo-SCT) in T-cell lymphomas: a French national survey from the Société Française de Greffe de Moelle et de Thérapie Cellulaire (SFGM-TC). J Clin Oncol. 2007;25:8095.

Howlader N, Noone A, Krapcho M, Miller D, et al., editors. SEER cancer statistics review, 1975-2014. Bethesda, MD: National Cancer Institute; 2017.
Kanate AS, Mussetti A, Kharfan-Dabaja MA, et al. Reduced-intensity transplantation for lymphomas using haploidentical related donors vs HLA-matched unrelated donors. Blood. 2016;127:938-47.

Lamy T, Damaj G, Soubeyran P, et al. R-CHOP 14 with or without radiotherapy in nonbulky limited-stage diffuse large B-cell lymphoma. Blood. 2018;131:174-81.

Neelapu SS, Locke FL, Go WY. CAR T-cell therapy in large B-cell lymphoma. N Engl J Med. 2018;378:1065.

Recher C, Coiffier B, Haioun C, et al. Intensified chemotherapy with ACVBP plus rituximab versus standard $\mathrm{CHOP}$ plus rituximab for the treatment of diffuse large B-cell lymphoma (LNH03-2B): an open-label randomised phase 3 trial. Lancet. 2011;378:1858-67.

Robinson SP, Boumendil A, Finel H, et al. High-dose therapy with BEAC conditioning compared to BEAM conditioning prior to autologous stem cell transplantation for non-Hodgkin lymphoma: no differences in toxicity or outcome. A matched-control study of the EBMT-Lymphoma Working Party. Bone Marrow Transplant. 2018. https://doi.org/10.1038/s41409018-0196-3. [Epub ahead of print].

Schmitz N, Nickelsen M, Ziepert M, et al. Conventional chemotherapy (CHOEP-14) with rituximab or highdose chemotherapy (MegaCHOEP) with rituximab for young, high-risk patients with aggressive B-cell lymphoma: an open-label, randomised, phase 3 trial (DSHNHL 2002-1). Lancet Oncol. 2012;13:1250-9.

Schmitz N, Ziepert M, Vitolo U, et al. The role of myeloablation for lymphoma. N Engl J Med. 2014;370:574-5.

Schuster SJ, Svoboda J, Chong EA, et al. Chimeric antigen receptor T cells in refractory B-cell lymphomas. $\mathrm{N}$ Engl J Med. 2017;377:2545-54.

Sehn LH, Berry B, Chhanabhai M, et al. The revised International Prognostic Index (R-IPI) is a better predictor of outcome than the standard IPI for patients with diffuse large B-cell lymphoma treated with R-CHOP. Blood. 2007;109:1857-61.

Sellner L, Boumendil A, Finel H, et al. Thiotepa-based high-dose therapy for autologous stem cell transplantation in lymphoma: a retrospective study from the EBMT. Bone Marrow Transplant. 2016;51:212-8.

Smith A, Crouch S, Lax S, et al. Lymphoma incidence, survival and prevalence 2004-2014: sub-type analyses from the UK's Haematological Malignancy Research Network. Br J Cancer. 2015;112:1575-84.

Staiger AM, Ziepert M, Horn H, et al. Clinical impact of the cell-of-origin classification and the MYC/ BCL2 dual expresser status in diffuse large B-cell lymphoma treated within prospective clinical trials of the German High-Grade Non-Hodgkin's Lymphoma Study Group. J Clin Oncol. 2017;35:2515-26.

Stiff PJ, Unger JM, Cook JR, et al. Autologous transplantation as consolidation for aggressive non-Hodgkin's lymphoma. N Engl J Med. 2013;369:1681-90.

Sureda A, Bader P, Cesaro S, et al. Indications for allo- and auto-SCT for haematological diseases, solid tumours 
and immune disorders: current practice in Europe, 2015. Bone Marrow Transplant. 2015;50:1037-56.

Swerdlow SH, Campo E, Harris NL et al. WHO classification of tumours of haematopoietic and lymphoid tissues. 4th ed (revised). Lyon: IARC; 2017.

Thieblemont C, Briere J, Mounier N, et al. The germinal center/activated B-cell subclassification has a prognostic impact for response to salvage therapy in relapsed/refractory diffuse large B-cell lymphoma: a bio-CORAL study. J Clin Oncol. 2011;29: 4079-87.

van Kampen RJ, Canals C, Schouten HC, et al. Allogeneic stem-cell transplantation as salvage therapy for patients with diffuse large B-cell non-Hodgkin's lym- phoma relapsing after an autologous stem-cell transplantation: an analysis of the European Group for Blood and Marrow Transplantation Registry. J Clin Oncol. 2011;29:1342-8.

Zhou Z, Sehn LH, Rademaker AW, et al. An enhanced International Prognostic Index (NCCN-IPI) for patients with diffuse large B-cell lymphoma treated in the rituximab era. Blood. 2014;123:837-42.

Ziepert M, Hasenclever D, Kuhnt E, et al. Standard International prognostic index remains a valid predictor of outcome for patients with aggressive CD20+ B-cell lymphoma in the rituximab era. J Clin Oncol. 2010;28:2373-80.

Open Access This chapter is licensed under the terms of the Creative Commons Attribution 4.0 International License (http://creativecommons.org/licenses/by/4.0/), which permits use, sharing, adaptation, distribution and reproduction in any medium or format, as long as you give appropriate credit to the original author(s) and the source, provide a link to the Creative Commons license and indicate if changes were made.

The images or other third party material in this chapter are included in the chapter's Creative Commons license, unless indicated otherwise in a credit line to the material. If material is not included in the chapter's Creative Commons license and your intended use is not permitted by statutory regulation or exceeds the permitted use, you will need to obtain permission directly from the copyright holder. 